\title{
Aspergilosis nasosinusal con extensión intracraneal en una paciente inmunocompetente: Una entidad infrecuente con características radiológicas típicas
}

\author{
Dres. Alberto Pons $E^{(1)}$, Sara Castanyer LI(2), Noemí Vidal $\mathbf{S}^{(3)}$, Julia Seul(4), Carles Aguilera G(5).
}

1. Servicio de Radiología, Hospital Universitari de Bellvitge. Barcelona, España.

2. Servicio de Radiología, IDI Hospital Universitari de Bellvitge. Barcelona, España.

3. Servicio de Anatomía Patológica, Hospital Universitari de Bellvitge. Barcelona, España.

4. Department of Radiology, Brigham and Women's Hospital, Boston. Massachusetts, USA.

5. Servicio de Radiología, IDI Hospital Universitari de Bellvitge. Barcelona, España.

Sinonasal aspergillosis with intracranial extension in an immunocompetent patient: a rare disease with typical imaging features

\begin{abstract}
Aspergillus is a ubiquitous fungus. The most common primary sites of infection are the respiratory tract and sinuses. Intracranial infection is rare and implies a high mortality. It occurs mainly by hematogenous extension from the lung, but in immunocompetent patients, direct extension from the sinuses is more common. We describe the case of a 25 year old woman from India who consulted in the emergency room of our hospital with chronic and progressive frontal headache. The findings in imaging studies suggested the diagnosis of fungal sinusitis with intracranial extension, being the most common pathogen of AspergiIlus. The diagnosis was anatomically-pathologically confirmed. We review the typical radiological findings which should help in the early diagnosis of this rare but potentially fatal disease.

Keywords: Abscess, Angioinvasivo, CT scan, Esthesioneuroblastoma, Fungal disease, Granuloma, Hematogenous extension, Immunocompromised, Immunocompetent, Immunosuppressed, Lymphoma, Magnetic resonance, Meningioma, Sinonasal disease Aspergillus, Sinusitis.
\end{abstract}

Resumen: El aspergilo es un hongo ubicuo. Las localizaciones de infección primaria más comunes son el tracto respiratorio y los senos paranasales. La afectación intracraneal es rara y conlleva una alta mortalidad. Ocurre mayoritariamente por extensión hematógena desde el pulmón, pero en pacientes inmunocompetentes, la extensión directa desde los senos paranasales es más común. Describimos el caso de una mujer de 25 años originaria de India que se presentó en el servicio de urgencia de nuestro centro hospitalario con cefalea frontal crónica y progresiva. Los hallazgos en los estudios de imágenes sugirieron el diagnóstico de sinusitis fúngica con extensión intracraneal, siendo el patógeno más frecuente el aspergilo. El diagnóstico fue confirmado anátomo-patológicamente. Revisamos los hallazgos radiológicos típicos que deben ayudar al diagnóstico precoz de esta entidad, rara, pero potencialmente mortal.

Palabras clave: Absceso, Angioinvasivo, Aspergillus, Estesioneuroblastoma, Extensión hematógena, Granuloma, Inmunocompetente, Inmunocomprometido, Inmunosuprimido, Linfoma, Meningioma, Patología fúngica, Patología sinonasal, Resonancia magnética, Sinusitis, Tomografía computarizada.

Pons $A$, et al. Aspergilosis nasosinusal con extensión intracraneal en una paciente inmunocompetente: una entidad infrecuente con características radiológicas típicas. Rev Chil Radiol 2014; 20(3): 116-121.

Contacto: Albert Pons E. / aponse@hotmail.com

Trabajo recibido el 08 de septiembre de 2013. Aceptado para publicación el 24 de junio de 2014.

\section{Caso clínico}

Una mujer de 25 años de edad acudió a la urgencia de nuestro centro por un cuadro de cefalea frontal progresiva de tres meses de duración y re- ciente aparición de náuseas y diplopía. El resto de la anamnesis, el examen físico y los test rutinarios de laboratorio no aportaron otra información clínica destacable. 


\section{Hallazgos radiológicos}

La tomografía computarizada (TC) craneal puso en evidencia la presencia de una masa intracerebral frontal izquierda espontáneamente densa y con moderado edema perilesional. Las celdillas etmoidales y el seno esfenoidal estaban ocupados por material de densidad de partes blandas con calcificaciones. También fue visible una dehiscencia de la lámina cribiforme (Figura 1).

La posterior resonancia magnética (RM) confirmó la masa frontal izquierda y puso de manifiesto su extensión desde la fosa nasal homolateral hasta el cuerpo calloso. Era isointensa en secuencias potenciadas en T1 y marcadamente hipointensa en T2 y FLAIR (Figura 2). No tenía restricción de la difusión ni incremento de la perfusión. La espectroscopia demostraba un aumento de colina (Cho), una reducción de creatinina $(\mathrm{Cr})$ y de $\mathrm{N}$-acetil-aspartato (NAA) en ambos tiempos de eco (TE) corto y largo (Figura 3).

\section{Manejo}

Se realizó una biopsia endoscópica de la porción intranasal de la lesión que demostró tejido inflamatorio sin evidencia de neoplasia. Dada la inespecificidad de los resultados, se procedió a realizar una biopsia abierta del componente lesional intracraneal. El estudio histológico reveló la presencia de inflamación granulomatosa por aspergilo (Figura 4). El tratamiento definitivo consistió en resección subtotal de la masa combinado con una larga pauta de amfotericina.

\section{Seguimiento}

La paciente no experimentó complicaciones posteriores a la cirugía y fue dada de alta 4 semanas después con indicación de amfotericina oral. En estas 4 semanas, hubo una importante mejoría en todos sus síntomas. No pudimos obtener imágenes de seguimiento dado que la paciente regresó a su país.

\section{Discusión}

El aspergilo es un hongo ubicuo. La localización más frecuente de primoinfección es el tracto respiratorio, seguido por las fosas nasales y senos paranasales ${ }^{(1-4)}$. Ha sido sugerido en la literatura un origen endémico en India, Sudan, Arabia Saudí y algunos países europeos del este ${ }^{(1,2)}$.

La aspergilosis es la causa más común de sinusitis fúngica tanto de carácter invasivo como no-invasivo. Siddiqui y Cols. ${ }^{(3)}$ describen cinco patrones de presentación con importantes implicaciones pronósticas. Son de más a menos frecuente y de menos a más grave:

- Bola de hongos o micetoma: generalmente afecta un solo seno (más frecuentemente maxilar) y a individuos no atópicos. Suele presentarse en los estudios por imágenes como una ocupación densa del seno comprometido con signos de sinusitis crónica y fenómenos de erosión y remodelación ósea.

- Sinusitis fúngica alérgica: suele afectar a individuos jóvenes atópicos con pansinusitis que condiciona expansión y adelgazamiento de las paredes de los senos afectados.
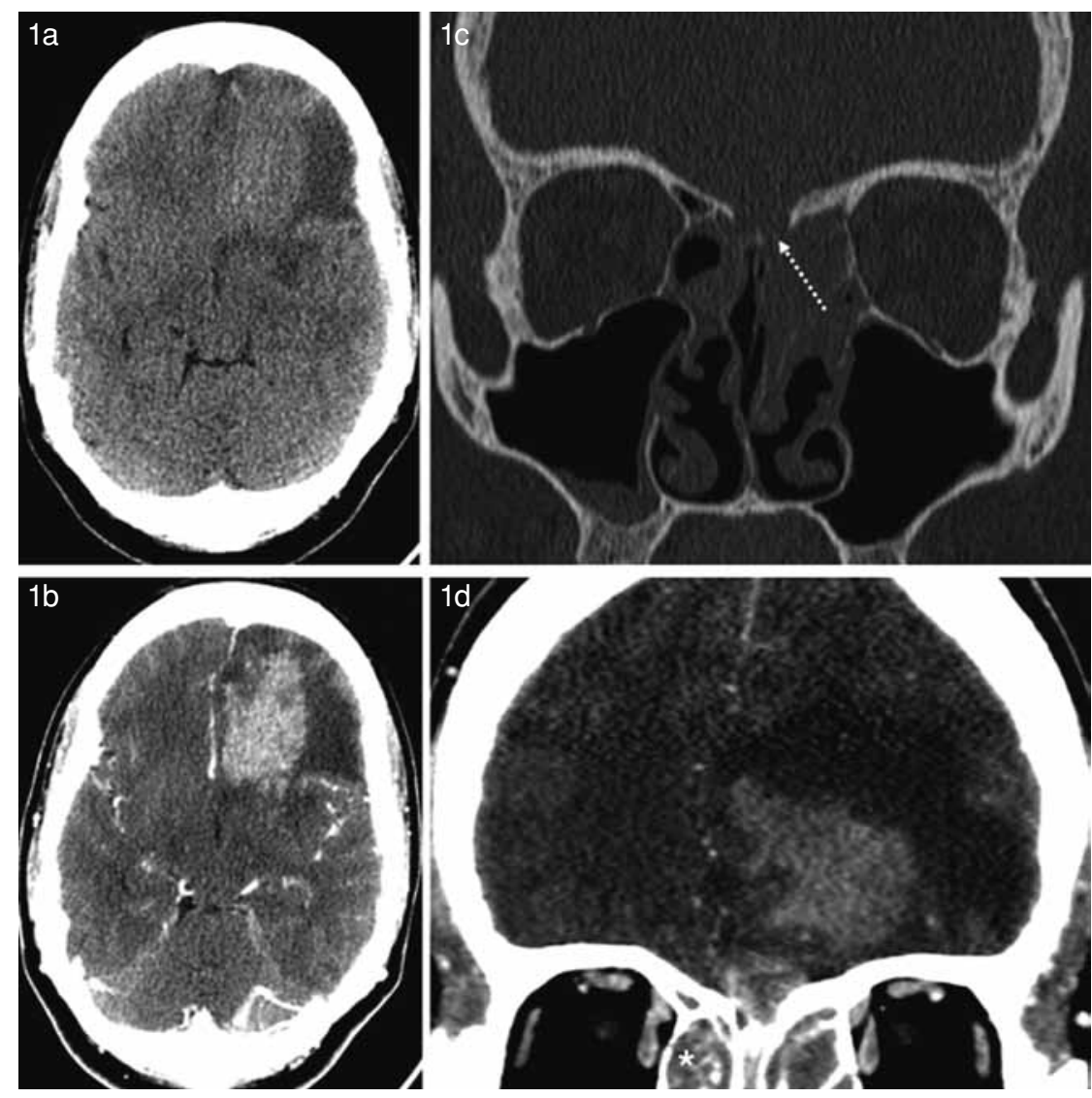

Figura 1. Tomografía computarizada. Imágenes axiales pre- (a) y postcontraste (b) que muestran una masa espontáneamente hiperdensa y ávidamente captante de contraste, de márgenes mal definidos e irregulares, en el lóbulo frontal izquierdo. Imágenes coronales en ventana de hueso (c) y de partes blandas (d) que revelan dehiscencia ósea en la lámina cribiforme (flecha discontinua) y ocupación parcial de la fosa nasal con calcificaciones (asterisco). 

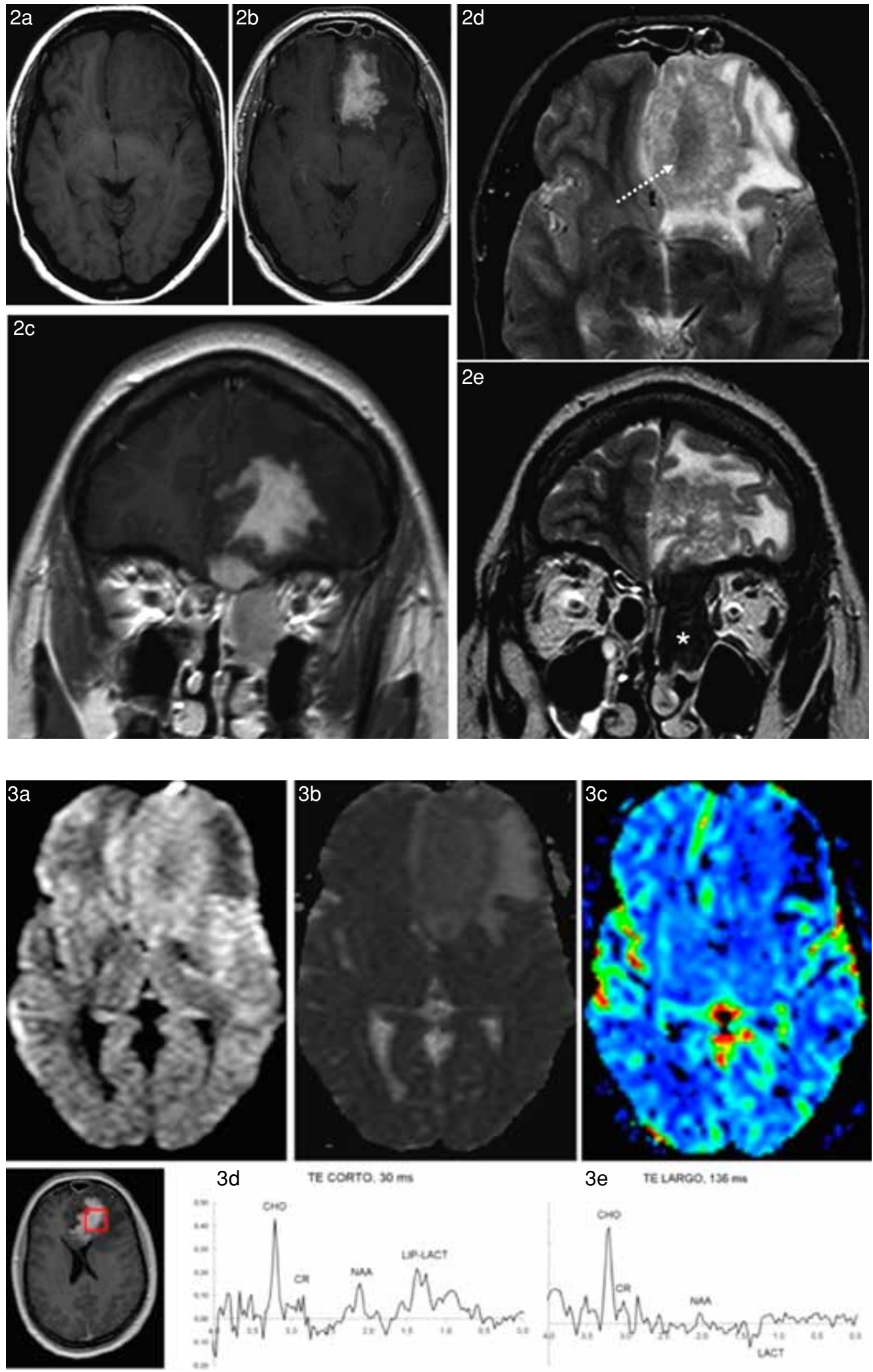

$3 d$
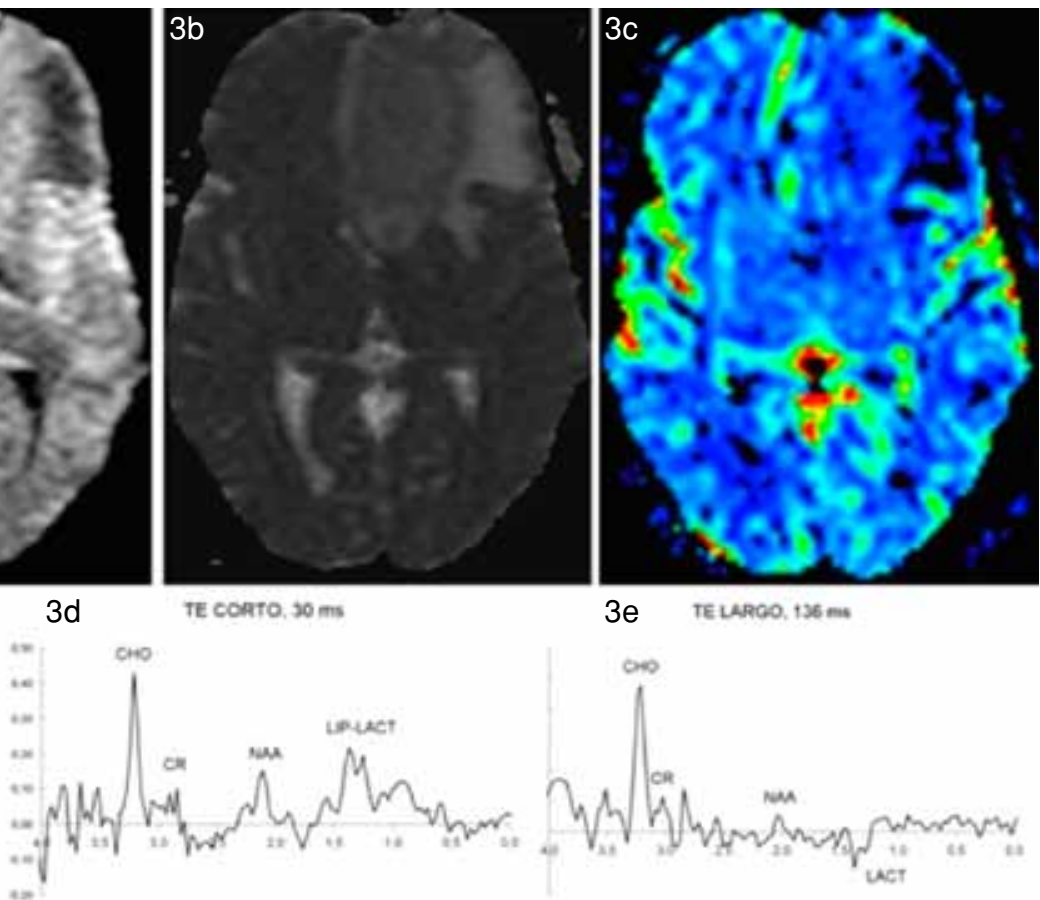

3e TEUACO, $136 \mathrm{~m}$
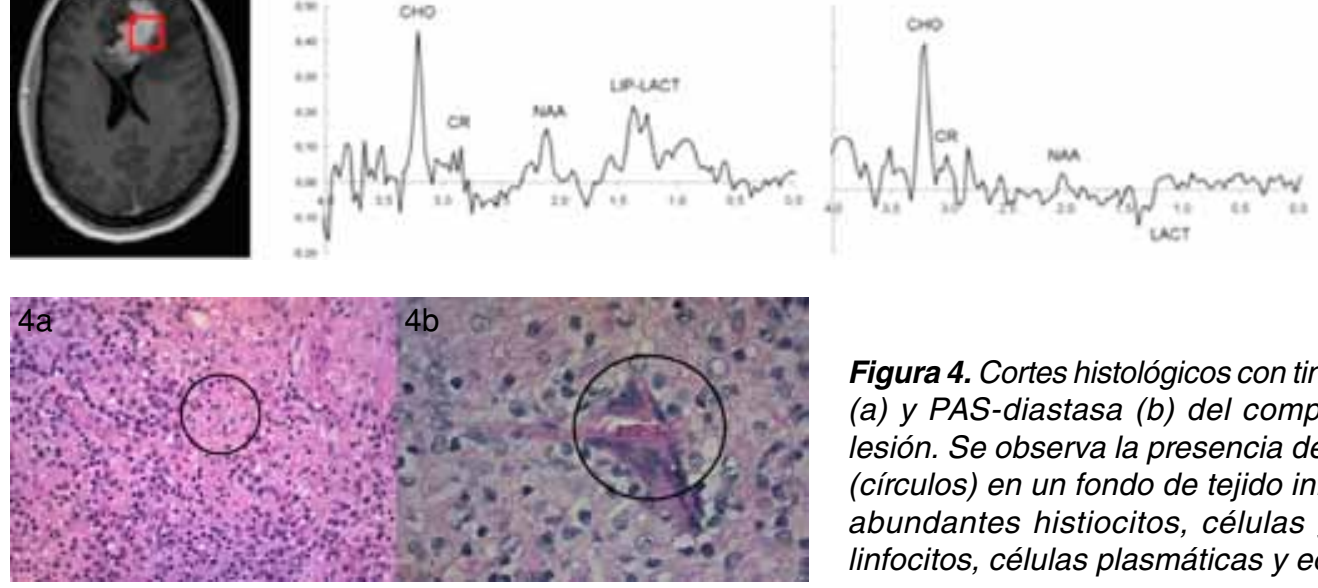

Figura 4. Cortes histológicos con tinciones hematoxilina-eosina (a) y PAS-diastasa (b) del componente intracraneal de la lesión. Se observa la presencia de hifas septadas y conidias (círculos) en un fondo de tejido inflamatorio hipercelular con abundantes histiocitos, células gigantes multinucleadas, linfocitos, células plasmáticas y eosinófilos. 
- Sinusitis fúngica crónica invasiva y sinusitis granulomatosa fúngica crónica invasiva: presentan un curso clínico larvado de lenta progresión y en los estudios de imagen pueden simular neoformaciones agresivas.

- Sinusitis fúngica aguda invasiva: afecta pacientes inmunocomprometidos y la invasión orbitaria, de base de cráneo e intracraneal es frecuente ${ }^{(5)}$.

La extensión intracraneal es una complicación infrecuente con altos rangos de mortalidad (13-50\%). Se ve más en pacientes inmunosuprimidos secundario a siembra hematógena desde una infección pulmonar primaria $^{(1-4)}$. En pacientes inmunocompetentes, la aspergilosis intracraneal es extremadamente rara (aproximadamente 30 casos en nuestra revisión de la literatura) y normalmente tiene lugar vía extensión directa de una sinusitis fúngica crónica invasiva ${ }^{(1,3,5,6)}$.

Las manifestaciones clínicas más frecuentes de la aspergilosis intracraneal reflejan la naturaleza angioinvasiva del microorganismo e incluyen meningitis, cerebritis, encefalitis, abscesos, infartos, sangrados y aneurismas micóticos ${ }^{(1-4,5)}$.

Las posibilidades diagnósticas de una masa intracraneal asociada a aspergilosis incluyen el granuloma y el absceso (Tabla II). Los abscesos aspergilosos ocurren de manera casi exclusiva en pacientes inmunosuprimidos y habitualmente son secundarios a diseminación hematógena o a extensión directa de una sinusitis fúngica aguda invasiva. Contrariamente, en pacientes inmunocompetentes, son más frecuentes las formaciones granulomatosas (por la propia respuesta inmune preservada del huésped) y normalmente se producen por extensión directa de una sinusitis fúngica crónica invasiva ${ }^{(1-4,5)}$.

El caso que motiva esta revisión representa una sinusitis granulomatosa fúngica crónica invasiva con extensión intracraneal en una paciente inmunocompetente.

La presentación clínica de las lesiones granulomatosas fúngicas intracraneales suele ser inespecífica. La mayoría de los pacientes permanecen afebriles y con síntomas neurológicos sutiles (por la lenta y larvada progresión de la enfermedad "per se"). Por ello, la sospecha diagnóstica por imágenes se vuelve muy importante siendo deseable que los radiólogos conozcan la entidad, para que, enfrentados a los hallazgos, puedan sugerirla oportunamente, lo que será determinante para el porvenir de la enfermedad. Las lesiones fúngicas granulomatosas intracraneales generalmente se tratan de manera combinada quirúrgica y medicamente ${ }^{(1-4)}$.

Tabla I. Resumen de diagnósticos diferenciales.

\begin{tabular}{|c|c|c|c|c|}
\hline $\begin{array}{l}\text { Patología/ } \\
\text { características }\end{array}$ & $\begin{array}{l}\text { Granuloma } \\
\text { fúngico }\end{array}$ & Linfoma & Estesioneuroblastoma & Meningioma \\
\hline Etiología & $\begin{array}{l}\text { Infeccioso. Forma } \\
\text { hifal del hongo }\end{array}$ & $\begin{array}{l}\text { Tumoral. Origen } \\
\text { hematológico }\end{array}$ & $\begin{array}{l}\text { Tumoral. Origen en } \\
\text { nervio olfatorio }\end{array}$ & $\begin{array}{l}\text { Tumoral. Origen } \\
\text { meningeo }\end{array}$ \\
\hline Sexo & Sin predilección & Varones & Sin predilección & Mujeres 2:1 \\
\hline Edad & Sin predilección & $50-60 a$ & Bimodal: $10-20$ a y $50-60 a$ & $40-60 a$ \\
\hline Incidencia & Rara & $\begin{array}{l}\text { Enfermedad frecuente/ } \\
\text { localización infrecuente } \\
(1,5-2 \%)\end{array}$ & Rara & $\begin{array}{l}\text { Muy frecuente ( } 20 \% \text { de } \\
\text { todos los tumores } \\
\text { intracraneales). } \\
\text { Localización atípica (10\%). }\end{array}$ \\
\hline $\begin{array}{l}\text { Factores de } \\
\text { riesgo }\end{array}$ & $\begin{array}{l}\text { Inmunosupresión y } \\
\text { zonas endémicas }\end{array}$ & Inmunosupresión & No & $\begin{array}{l}\text { Radiación y NFII } \\
\text { (especialmente si <40a) }\end{array}$ \\
\hline Tratamiento & Médico+quirúrgico & $\begin{array}{l}\text { Radioterapia+ } \\
\text { Quimioterapia }\end{array}$ & $\begin{array}{l}\text { Cirugía/ } \\
\text { Radioterapia }\end{array}$ & $\begin{array}{l}\text { Observación vs } \\
\text { Cirugía/Radioterapia }\end{array}$ \\
\hline Pronóstico & $\begin{array}{l}\text { Altas tasas de } \\
\text { mortalidad (13-50\%) }\end{array}$ & $\begin{array}{l}70 \% \text { libre de } \\
\text { enfermedad a los } 5 \text { años }\end{array}$ & $\begin{array}{l}\text { 75-77\% superviviencia } \\
\text { a } 5 \text { años }\end{array}$ & Excelente \\
\hline $\begin{array}{l}\text { Claves } \\
\text { diagnósticas } \\
\text { por imagen }\end{array}$ & $\begin{array}{l}\text { Masa hipointensa en } \\
\text { T2 de márgenes } \\
\text { infiltrativos y con } \\
\text { origen en fosas nasales } \\
\text { o senos paransales }\end{array}$ & $\begin{array}{l}\text { Abscencia de necrosis } \\
\text { Restricción de la } \\
\text { difusión }\end{array}$ & $\begin{array}{l}\text { Quistes periféricos en } \\
\text { el margen craneal del } \\
\text { tumor }\end{array}$ & $\begin{array}{l}\text { Base dural (cola dural), } \\
\text { Hiperostosis. Calcificaciones } \\
\text { y realce interno } \\
\text { homogéneo }\end{array}$ \\
\hline
\end{tabular}


Tabla II. Resumen de manifestaciones de la aspergilosis intracraneal.

\begin{tabular}{|c|c|}
\hline Tipo de aspergilosis intracraneal & Características generales \\
\hline Forma angioinvasiva & $\begin{array}{l}\text { - Siempre origen en diseminación hematógena. } \\
\text { - Pacientes inmunosuprimidos. } \\
\text { Imagen: meningitis, encefalitis, infartos, hemorragias, seudoaneurismas. }\end{array}$ \\
\hline Absceso & $\begin{array}{l}\text { - Origen en diseminación hematógena o extensión directa de sinusitis aguda invasiva. } \\
\text { - Pacientes inmunosuprimidos. } \\
\text { sus características proyecciones paciones pares centrípetas de la pared del absceso. }\end{array}$ \\
\hline Granuloma & $\begin{array}{l}\text { - Extensión directa de sinussitis crónica invasiva. } \\
\text { Tres patrones: 1- Masa intracraneal intracerebral, 2- Masa intracraneal extradural, } \\
\text { 3- Afectación de base de cráneo y/o órbita. } \\
\text { - Inmunosuprimidos e inmunocompetentes (especialmente en áreas endémicas). } \\
\text { - Imagen: hipointensidad en T2 (MG and FE necesarios para supervivencia del } \\
\text { hongo en su forma hifal). }\end{array}$ \\
\hline
\end{tabular}

Los hallazgos en la TC sugerentes de una lesión intracraneal fúngica granulomatosa incluyen la presencia de una masa espontáneamente hiperdensa que se realza ávida y uniformemente con el contraste y está en contigüidad a un seno paranasal ocupado, con calcificaciones y zonas de dehiscencia ósea ${ }^{(1,3,4)}$. A estas características se agregan en el estudio de RM como manifestaciones típicas: una masa de marcada hipointensidad central con un halo periférico irregular hiperintenso en secuencias $\mathrm{T} 2^{(1-3)}$, con estudios que muestran perfusión disminuida y una difusión facilitada ${ }^{(6)}$.

El centro hipointenso en T2 representa el hallazgo por imagen más característico y se debe a la presencia en la lesión de elementos paramagnéticos como hierro y magnesio. Estos iones metálicos son necesarios para el crecimiento del hongo en su forma hifal, el estado en que más frecuentemente se halla el aspergilo en los granulomas. La periferia hiperintensa en T2 corresponde a la reacción inflamatoria normal del huésped ${ }^{(1,2)}$.

En nuestra revisión de la literatura no encontramos referencias a un patrón espectroscópico específico para lesiones granulomatosas. De todos modos, el hecho de que exista sólo un incremento leve de colina en la lesión y en el área de edema circundante podría sugerir una lesión seudotumoral en vez de una lesión neoplásica ${ }^{(6)}$. En nuestro caso las relaciones Cho: Cr y Cho: NAA encontradas sugirieron de manera errónea una etiología tumoral ${ }^{(7)}$.

Si bien el caso presentado pone en evidencia hallazgos radiológicos típicos de una masa granulomatosa fúngica, en el diagnóstico diferencial se deben considerar otras entidades que pueden presentar extensión intracraneal desde las fosas nasales y senos paranasales o a la inversa (Tabla I). Entre ellas, en particular, el estesioneuroblastoma, el linfoma y el meningioma.

El estesioneuroblastoma es una neoplasia rara de la familia de los PNET que se origina en el nervio olfatorio. En los estudios por imagen aparece como una masa de morfología en "reloj de arena" con su porción superior en fosa craneal anterior y la inferior en fosas nasales. La presencia de quistes periféricos en la vertiente craneal o interfase tumor-cerebro se ha descrito como característico de este tumor.

El linfoma infrecuentemente afecta la cavidad naso-sinusal y cuando lo hace suele afectar a varones de edad avanzada. Los estudios de imagen con frecuencia muestran una masa homogénea, densa en la TC, con señal intermedia-baja en T2 y marcada restricción de la difusión debido a su hipercelularidad.

El meningioma del surco olfatorio se presenta como un tumor expansivo, extra-axial, de base dural. Sus rasgos por imagen incluyen hiperdensidad espontánea y calcificaciones en la TC, realce homogéneo e hiperostosis del hueso adyacente. En la RM suelen tener señal intermedio-bajo en T2 y la espectroscopia muestra característicamente presencia de alanina $(1.3-1.5 \mathrm{ppm})^{(9-11,13)}$.

Otras entidades malignas como el carcinoma nasofaríngeo o el carcinoma de etmoides se manifestarían con importante destrucción ósea en lugar de erosión y/o remodelación. Consideraciones diagnósticas adicionales raras incluirían la granulomatosis de Wegener, la sarcoidosis y el seudotumor inflamatorio. La extensión intracraneal de estas últimas entidades ha sido descrita, pero las imágenes son poco específicas y su diagnóstico suele ser por exclusión ${ }^{(9,14,15)}$. 
A modo de conclusión: ante la presencia de una lesión intracraneal expansiva, hiperdensa en la TC, de centro hipointenso en secuencias T2 de RM y localizada en contigüidad a un seno paranasal ocupado y con calcificaciones, debemos considerar el diagnóstico de sinusitis granulomatosa crónica invasiva con extensión intracraneal, siendo el aspergilo el patógeno más frecuente. Es deseable que los radiólogos conozcan esta entidad y sus variantes, ya que se considera una enfermedad emergente dada la prevalencia del SIDA (síndrome de inmunodeficiencia humana adquirida) y de la creciente inmigración en todo el mundo.

\section{Bibliografía}

1. Phuttharak W, Hesselink JR, Wixom C. MR Features of Cerebral Aspergillosis in an Immunocompetent Patient: Correlation with Histology and Elemental Analysis. AJNR Am J Neuroradiol 2005; 26: 835-838.

2. Turgut M, O'zsunar Y, O'ncq S, Akyqz O, Ertugrul M, et al. Invasive fungal granuloma of the brain caused by Aspergillus fumigatus: a case report and review of the literature. Surgical Neurology 2008; 69: 169-174.

3. Siddiqui AA, Bashir SH, Ali Shah A, Sajjad Z, Ahmed $N$, et al. Diagnostic MRI Imaging features of craniocerebral aspergillosis of sino-nasal origin in inmunocompetent patients. Acta Neurochir 2006; 148: 155-166.

4. Luthra G, Parihar A, Nath K, Jaiswal S, Prasad KN, et al. Comparative Evaluation of Fungal, Tubercular, and Pyogenic Brain Abscesses with Conventional and Diffusion MR Imaging and Proton MR Spectroscopy. AJNR Am J Neuroradiol 2007; 28: 1332-1338.

5. Aribandi M, McCoy V, Bazan III C. Imaging Features of
Invasive and Noninvasive Fungal Sinusitis: A Review. RadioGraphics 2007; 27: 1283-1296.

6. Jitender Saini, Arun Kumar Gupta, Milan Babulal Jolapara, Somenath Chatterjee, Hima S. Pendharkar, Kesavadas Chandreshekher, et al. Imaging Findings in Intracranial Aspergillus Infection in Immunocompetent Patients. World Neurosurgery 2010; 74: 661-670.

7. Majós C, Aguilera C, Alonso J, Julià-Sapé M, Castanyer S, et al. Proton MR Spectroscopy Improves Discrimination between Tumor and Pseudotumoral Lesion in Solid Brain Masses. AJNR AM J Neuroradiol 2009; 30: 544-551.

8. Slone HW, Blake JJ, Shah R, et al. CT and MRI findings of intracranial lymphoma. AJR Am J Roentgenol 2005; 184: 1679-1685.

9. Harnsberger HR, Glastonbury CM, Michel MA, Koch BL. Diagnostic Imaging: Head and Neck. Lippincott Williams \& Wilkins (2nd edition, 2011).

10. Osborn AG. Brain: Imaging, Pathology, and Anatomy. Lippincott Williams \& Wilkins (2012).

11. Grossman RI, Yousem DM. Neuroradiology, the requisites. Mosby Inc. (2003).

12. Rosengren JE, Jing BS, Wallace S. Radiographic features of olfactory neuroblastoma. AJR Am J Roentgenol 1979; 132: 945-948.

13. Buetow MP, Buetow PC, Smirniotopoulos JG. From the Archives of the AFIP: Typical, Atypical, and Misleading Features in Meningioma. Radiographics 1991; 11: 1087-1106.

14. OY Dessouky. Isolated sinonasal sarcoidosis with intracranial extension: case report. Acta Otorhinolaryngol Ital 2000; 28: 306-308.

15. Sung Bin Park, Jeong Hyun Lee and Young Cheol Weon. Imaging Findings of Head and Neck Inflammatory Pseudotumor. AJR Am J Roentgenol 2009; 193(4): 1180-1186. 